\title{
Dynamics and thermalization of a Bose-Einstein condensate in a Sinai-oscillator trap
}

\author{
Leonardo Ermann, ${ }^{1,2}$ Eduardo Vergini, ${ }^{1}$ and Dima L. Shepelyansky ${ }^{3}$ \\ ${ }^{1}$ Departamento de Física, Gerencia de Investigación y Aplicaciones, Comisión Nacional de Energía Atómica. \\ Av. del Libertador 8250, 1429 Buenos Aires, Argentina \\ ${ }^{2}$ CONICET, Godoy Cruz 2290 (C1425FQB) CABA, Argentina \\ ${ }^{3}$ Laboratoire de Physique Théorique, IRSAMC, Université de Toulouse, CNRS, UPS, 31062 Toulouse, France
}

(Received 9 May 2016; published 25 July 2016)

\begin{abstract}
We study numerically the evolution of Bose-Einstein condensate in the Sinai-oscillator trap described by the Gross-Pitaevskii equation in two dimensions. In the absence of interactions, this trap mimics the properties of Sinai billiards where the classical dynamics is chaotic and the quantum evolution is described by generic properties of quantum chaos and random matrix theory. We show that, above a certain border, the nonlinear interactions between atoms lead to the emergence of dynamical thermalization which generates the statistical Bose-Einstein distribution over eigenmodes of the system without interactions. Below the thermalization border, the evolution remains quasi-integrable. Such a Sinai-oscillator trap, formed by the oscillator potential and a repulsive disk located in the vicinity of the center, had been already realized in first experiments with the Bose-Einstein condensate formation by Ketterle group in 1995 and we argue that it can form a convenient test bed for experimental investigations of dynamical of thermalization. Possible links and implications for Kolmogorov turbulence in absence of noise are also discussed.
\end{abstract}

DOI: 10.1103/PhysRevA.94.013618

\section{INTRODUCTION}

One of the first experimental realizations of Bose-Einstein condensate (BEC) has been done with sodium atoms trapped in a novel trap that employed both magnetic and optical forces [1]. In this trap, the repulsive optical potential is created by tightly focusing an intense blue-detuned laser that generates a repulsive optical plug bunging a hole in a center of magnetic trap where nonadiabatic spin flips lead to a loss of atoms. Further developments of BEC traps, and remarkable progress of BEC experiments theory, are reviewed in [2-5].

In spite of these achievements, the fundamental question about interplay of dynamics, interactions, and thermalization of BEC in a concrete trap configuration still awaits clarification. In this work, we address this question in the frame of the Gross-Pitaevskii equation (GPE) [4,5] for the two-dimensional (2D) version of the trap used in the experimental setup [1]. Thus, the trap potential is represented by a $2 \mathrm{D}$ harmonic potential and a rigid disk which center is located in a vicinity of the center of harmonic potential. If the harmonic potential is replaced by rigid walls forming a square or rectangle, then the classical dynamics in such a Sinai billiard is proven to be completely chaotic [6]. The recent analysis of the trap with the walls formed by a harmonic potential shows that the dynamics remains chaotic with a very small measure of integrable dynamics [7]. This trap was called the Sinai oscillator [7] due to a similarity with a Sinai billiard. Since the realization of rigid walls is rather difficult for experimental realization, the case of Sinai-oscillator trap becomes much more attractive for combined theoretical and experimental investigations. In fact, a Sinai-oscillator trap in three dimensions (3D) had been implemented in [1]. Here, we restrict our investigations to the 2D case expecting that its main features will be preserved in 3D.

The quantum properties of Sinai oscillator are characterized within random matrix theory [9] by the Wigner-Dyson statistics of energy levels [8]. The properties of the eigenstates are typical for those of systems of quantum chaos and now are well understood (see, e.g., [10,11]).

Below we show that the Sinai-oscillator trap in $2 \mathrm{D}$ is also characterized by the properties of quantum chaos: the quantum eigenstates of Sinai oscillator are ergodic and the level spacing statistic is described by the random matrix theory, in agreement with the Bohigas-Giannoni-Schmit conjecture $[8,10]$. However, still there is no thermalization in this system since the eigenstates are preserved in absence of interactions. Thus, in this work we analyze the dynamics and thermalization conditions for BEC in the Sinai oscillator in the frame of the GPE equation. The GPE description is valid in the regime where the BEC temperature $T$ is below the critical temperature of Bose-Einstein condensation $T_{c}$ [12] and when the validity of GPE description is well justified [4,5].

Even though from the mathematical viewpoint the question about existence of solutions of the GPE in such a trap, at moderate nonlinearity and large times, remains an open problem (see, e.g., $[13,14]$ ). Indeed, the GPE can be rewritten in the basis of linear eigenstates (modes) where the coupling between modes takes place only due to the nonlinearity in GPE. In this representation, each mode can be considered as an independent oscillator degree of freedom and in case of thermalization, induced by nonlinearity, one should expect energy equipartition over all modes [12] leading to ultraviolet catastrophe and energy transfer to high-energy modes. In fact, the Planck constant and the Planck law had been introduced for a black-body radiation to avoid such a divergence [15]. However, the Planck distribution is valid for quantum systems, while in our case of the GPE Sinai oscillator there is no second quantization. Thus, due to classical nonlinear interactions between modes, one would expect to have a classical ergodicity with equipartition of energy between modes.

Indeed, such an equipartition expectation was at the origin of the studies of the Fermi-Pasta-Ulam (FPU) problem [16,17]. Nevertheless, its absence is consequence of the proximity to the integrable Toda lattice (see, e.g., [18,19] and references 
therein). Thus, the FPU oscillator chain has certain specific features which break system ergodicity in energy. However, it is natural to expect that in a generic case, when eigenstates of a linear system are ergodic and dynamical chaos of classical trajectories takes place, the energy equipartition over modes should appear above certain border of nonlinear interaction strength between modes.

In spite of these expectations of energy equipartition over modes, the recent studies of the GPE in Bunimovich stadium showed that the nonlinearity produces an effective dynamical thermalization in a completely isolated system, without any contact with external thermal bath, with the probabilities over linear modes described by the Bose-Einstein (BE) distribution [7]. Thus, the probabilities on high-energy modes drop rapidly and the ultraviolet catastrophe is absent. An experimental realization of the Bunimovich billiard with cold atoms is possible but is not so simple. Due to this reason, we consider here the GPE Sinai-oscillator trap which in fact has been already built in [1-3] but without investigation of phenomenon of dynamical thermalization. Our results show the presence of dynamical thermalization with BE distribution in this system even if some aspects still should be clarified for time evolution of very large time scales.

The model description and the quantum chaos features of linear system are described in Sec. II. The thermalization equations and the formalism are presented in Sec. III. The obtained numerical results for the GPE evolution are presented and discussed in Sec. IV. Numerical methods and behavior on large time scales are discussed in Sec. V. The discussion of the main results is presented in Sec. VI.

\section{MODEL DESCRIPTION AND QUANTUM CHAOS PROPERTIES}

The dynamics of the classical Sinai oscillator is described by the Hamiltonian

$$
H=\frac{1}{2 m}\left(p_{x}^{2}+p_{y}^{2}\right)+\frac{m}{2}\left(\omega_{x}^{2} x^{2}+\omega_{y}^{2} y^{2}\right)+V_{d}(x, y),
$$

with the first two terms being 2D oscillator with frequencies $\omega_{x}, \omega_{y}$, while the last term describes the potential wall of elastic disk of radius $r_{d}$. In our studies, we fixed the mass $m=1$, frequencies $\omega_{x}=1, \omega_{y}=\sqrt{2}$, and disk radius $r_{d}=1$. The disk center is placed at $\left(x_{d}, y_{d}\right)=\left(-\frac{1}{2},-\frac{1}{2}\right)$ so that the disk bungs a hole in the center as it was the case in the experiments [1].

It is convenient to describe the classical dynamics on the Poincaré section using the canonical variables at the moment of the bounce with the disk. We take the phase $\varphi$, given by the angle measured from $x$ axis, and the conjugated dimensionless orbital momentum $\kappa=\sin \theta$, where $\theta$ is the angle of momentum $\vec{p}$ counted from the normal to the circle (see Fig. 1). Such a pair of conjugated variables represents a standard choice for the description of dynamics in billiards (see, e.g., [10,11]).

Figure 1 shows that almost all phase space, accessible at a given energy, is chaotic (see, e.g., [20,21] on properties of dynamical chaos). Only very tiny islands of regular motion are found at $E=3$ (practically not visible on the Poincaré section). At the energy $E=1.5$, the dynamics exists only
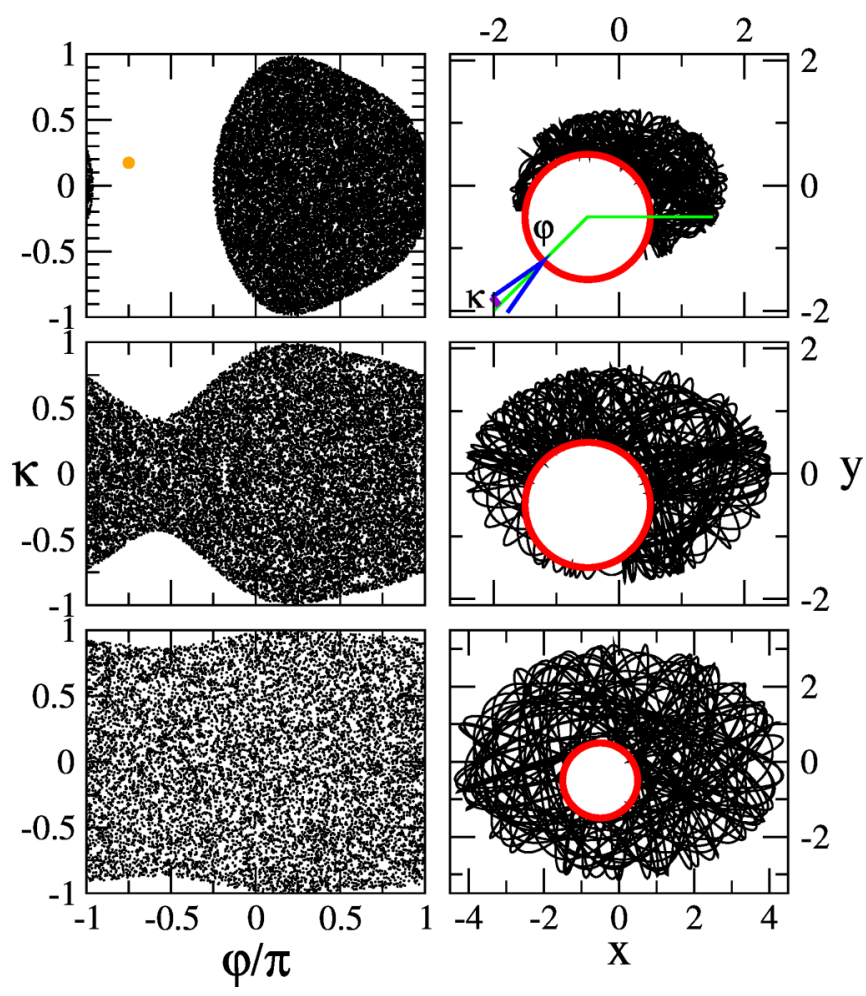

FIG. 1. Left panels show Poincaré section given by canonical variables $\varphi$ and $\kappa$, taken at the disk bounce; points show trajectories evolving up to time $t=10000$ with five random initial conditions at initial energies $E=1.5 ; 3 ; 10$ in top, center, and bottom panels, respectively. Right panels show dynamics of one trajectory in $(x, y)$ plane evolving up to time $t=300$ with initial energy as in left panels $E=1.5 ; 3 ; 10$ in top, center, and bottom panels, respectively. In the top right panel, an example of canonical variables (gray color) is shown with $\varphi=-0.75 \pi$ [green (gray) lines] and $\kappa=\sin \pi / 18$ [violet (black) lines], these variables are represented in the top left panel by the orange (gray) point. Disk border is shown in right panels by red (gray) circle.

on one side of the disk (variation of $\varphi$ is bounded, $-0.4<$ $\varphi / \pi \leqslant 1$ ) due to symmetry breaking of the system. At larger energies, the trajectories make complete rotations around the disk. The amplitude of oscillations grows with energy approximately in the same way as in a usual 2D oscillator in absence of disk. However, the scattering on disk makes the dynamics chaotic in a similar manner as for a standard Sinai billiard [6].

The BEC evolution in the Sinai oscillator trap is described by the GPE, which reads as

$$
\begin{aligned}
i \hbar \frac{\partial \psi(\vec{r}, t)}{\partial t}= & -\frac{\hbar^{2}}{2 m} \vec{\nabla}^{2} \psi(\vec{r}, t) \\
& +\left[\frac{m}{2}\left(\omega_{x}^{2} x^{2}+\omega_{y}^{2} y^{2}\right)+V_{d}(x, y)\right] \psi(\vec{r}, t) \\
& +\beta|\psi(\vec{r}, t)|^{2} \psi(\vec{r}, t) .
\end{aligned}
$$

Here in (2), we use the same oscillator and disk parameters as in (1) and take $\hbar=1$. The wave function is normalized to unity $W=\int|\psi(x, y)|^{2} d x d y=1$. Then, the parameter $\beta$ describes the nonlinear interactions of atoms in BEC. All 


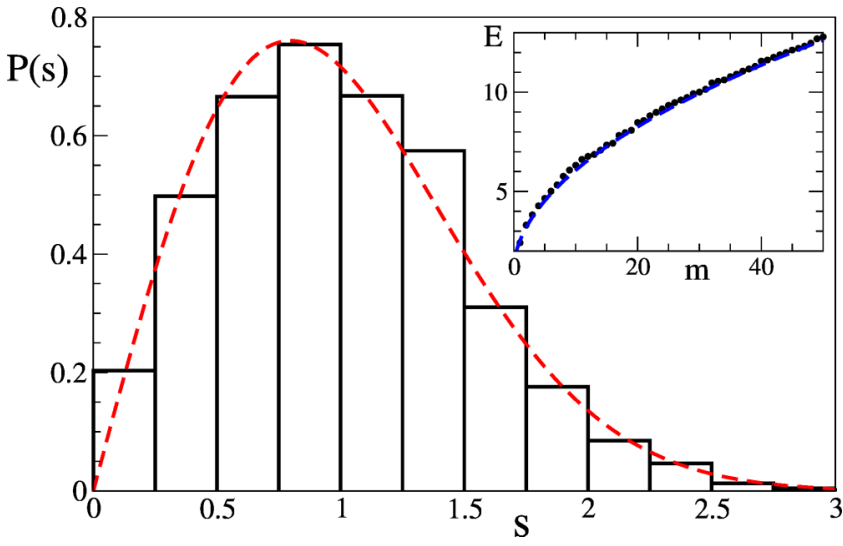

FIG. 2. Nearest-neighbor spacing distribution $P(s)$ for the first 2500 unfolded eigenenergies of the Sinai oscillator (1). The red dashed curve represents the Wigner surmise $P(s)=$ $(\pi s / 2) \exp \left(-\pi s^{2} / 4\right)$. Inset panel shows energy eigenvalues $E=E_{m}$ as a function of $m$ for the first 50 eigenvalues $1 \leqslant m \leqslant 50$. Dashed blue curve represents the theoretical Weyl law $m(E)=E^{2} /(2 \sqrt{2})-$ $E / 2$.

the results presented in the paper are expressed in these dimensionless units. Thus, the energy $E$ is expressed in units of $E_{u}=\hbar^{2} /\left(m r_{d}^{2}\right)=1$; the distance is measured in units of $r_{d}=1$; time is measured in units of $t_{u}=\hbar / E_{u}=1 ; \beta$ is measured in units of $\beta_{u}=1 / r_{d}^{2}=1$.

Since the classical dynamics is chaotic and the measure of integrable islands is very small, it is natural to expect that at zero nonlinearity $\beta=0$ the Sinai oscillator belongs to systems of quantum chaos $[8,10,11]$. Indeed, using the advanced methods of quantum chaos on numerical computation of eigenenergies and eigenstates in chaotic billiards (see, e.g., [22]), we find numerically several thousands of eigenenergies $E_{m}$ and eigenstates $\phi_{m}$ (linear modes) at $\beta=0$.

The level spacing statistics $P(s)$ for the first 2500 energy levels with the unfolding procedure (see, e.g., [10]) is shown in Fig. 2. The results are in good agreement with the Wigner surmise confirming the validity of the Bohigas-GiannoniSchmit conjecture $[8,10]$. The system energy $E_{m}$ grows with the level number $m$ in agreement with the Weyl law as it is shown in the inset of Fig. 2.

The linear eigenstates $\phi_{m}$ have a rather complex structure covering the accessible area in $(x, y)$ plane with chaotic fluctuations. The probability distributions in the $(x, y)$ plane are shown for first 100 eigenstates in [23]; in addition, some eigenstates are also shown below.

The GPE (2) can be also rewritten in the basis of linear eigenstates $\phi_{m}$ using the completeness of this basis and presenting the wave function by the expansion $\psi(x, y, t)=$ $\sum_{m} C_{m}(t) \phi_{m}(x, y)$, where $C_{m}(t)$ are time-dependent probability amplitudes in this basis. Then, in this basis the GPE reads as

$$
i \frac{\partial C_{m}}{\partial t}=E_{m} C_{m}+\beta \sum_{m_{1} m_{2} m_{3}} U_{m m_{1} m_{2} m_{3}} C_{m_{1}} C_{m_{2}}^{*} C_{m_{3}} .
$$

Here, the transitions between eigenmodes appear only due to the nonlinear $\beta$ term, and the transition matrix elements are

$$
U_{m m_{1} m_{2} m_{3}}=\int d x d y \phi_{m}^{*} \phi_{m_{1}} \phi_{m_{2}}^{*} \phi_{m_{3}} .
$$

In our case, in absence of a magnetic field, the eigenstates $\phi_{m}$ are real, but we keep the general expression valid also for complex eigenstates.

A similar type of representation (4) was used for the analysis of effects of nonlinearity on the Anderson localization in disordered lattices [24] known also as the DANSE model $[25,26]$. In this model, it was found that a moderate nonlinearity leads to a destruction of the Anderson localization of linear eigenmodes and a subdiffusive spreading of wave packet over lattice sites with time. Such a spreading has been studied by different groups (see, e.g., [24-28] and references therein). Even if the representations for the GPE Sinai-oscillator (4) and DANSE models are similar, there are significant differences: (a) in DANSE the eigenenergies are bounded in a finite-energy band while here $E_{m} \propto \sqrt{m}$ are growing with $m$ (we note that for the Bunimovich billiard we have $E_{m} \propto m$ [7]); (b) in DANSE the transitions $U_{m m_{1} m_{2} m_{3}}$ give coupling mainly between states inside the same localization length while here there are transitions even between very different $m$ values. At the same time, we should say that the properties of matrix elements $U_{m m_{1} m_{2} m_{3}}$ are still awaiting their detailed analysis for the cases of Sinai oscillator and Bunimovich billiard.

We also note that the question of energy transfer to high-energy modes has certain links with the Kolmogorov turbulence which is based on the concept of energy flow from large to small scales via the inertial interval (see [29,30] and references therein). The energy is injected at large scale and absorbed on small scales and a presence of some small noise is assumed to induce thermalization. In such an approach, the "quantum" turbulence in GPE [or nonlinear Schrödinger equation (NLS)] has been studied in a rectangular 2D billiard [31] and in 3D cube [32]. Due to the billiard shape chosen there, the ray dynamics is integrable and it is not obvious if the dynamical thermalization takes place in such a billiard in absence of noise. Below, we will see that at moderate nonlinearity and absence of noise there is no dynamical thermalization in oscillator trap and billiards of rectangular shape (see the later case in [7]). In fact, in purely dynamical systems (without external noise), it is possible that the Kolmogorov flow to high modes can be stopped by Kolmogorov-Arnold-Moser (KAM) integrability [20,21] and Anderson localization [33].

Our results show that the quantum chaos for linear eigenmodes facilitates onset of dynamic thermalization, appearing in an isolated system without any noise, when the strength of nonlinear term is above a certain dynamical thermalization border $\beta>\beta_{c}$.

\section{THERMODYNAMIC FORMALISM}

The dynamical thermalization in nonlinear chains with disorder has been studied in [34,35] where it was shown that the quantum Gibbs distribution appears in an isolated system above a certain border of nonlinearity $\beta>\beta_{c}$. The dynamical thermalization for the GPE in a chaotic Bunimovich billiard has been established in [7].

Below for a reader convenience we present the thermalization formalism which directly follows from the standard statistical description of Bose gas [12]. Indeed, we assume that the nonlinearity is moderate and that the nonlinear term 
provides a small energy shift which can be neglected. Then, the energy levels are those of the quantum Sinai oscillator with the usual quantum chaos properties and the energy levels $E_{m}$ at $\beta=0$. As the energy and the norm of the system are conserved for the quantum evolution of the system [Eq. (2)], the thermalization ansatz gives the steady-state probabilities $\rho_{m}$ on energy levels:

$$
\rho_{m}=1 /\left\{\exp \left[\left(E_{m}-E_{g}-\mu\right) / T\right]-1\right\},
$$

where $E_{g}=1.685$ is the energy of the ground state, $T$ is the temperature of the system, $\mu(T)$ is the chemical potential dependent on temperature. The parameters $T$ and $\mu$ are determined by the norm conservation $\sum_{m=1}^{\infty} \rho_{m}=1$ (we have only one particle in the system) and the initial energy $\sum_{m} E_{m} \rho_{m}=E$. The entropy $S$ of the system is determined by the usual relation [12] $S=-\sum_{m} \rho_{m} \ln \rho_{m}$. The relation (5), with normalization condition and equation of energy, determines the implicit dependencies on temperature $E(T), S(T), \mu(T)$.

As pointed out in $[7,34,35]$, the advantage of energy $E$ and entropy $S$ is that both are extensive variables, thus, they are self-averaging and due to that they have reduced fluctuations. Due to this feature $S$ and $E$ are especially convenient for verification of the thermalization ansatz (5) which gives the theoretical dependence $S(E)$ in the assumption that the dynamical thermalization emerges in the GPE Sinai oscillator due to dynamical chaos in absence of any external noise or thermostat.

\section{NUMERICAL RESULTS}

The numerical integration of GPE (2) follows the approach used for the Bunimovich billiard in [7]: we introduce a space grid with size $N_{s}=n_{x} \times n_{y}=201 \times 141=28.341$ spatial points. The time step is performed with the Trotter decomposition of linear and nonlinear terms with a time step $\Delta t=0.01$. Thus, the nonlinear term gives the wave-function transformation in coordinate space $\bar{\psi}(x, y)=$ $\exp \left[-i \Delta t \beta|\psi(x, y)|^{2}\right] \psi(x, y)$, which is then transformed from coordinate space to the linear eigenbasis $\phi_{m}$ (we use $N_{e}=$ 2000 linear eigenstates). The transformation from space grid to linear eigenfunction index $m$ is done via a precomputed transfer matrix $A_{j m}$ (here, $1 \leqslant j \leqslant N_{s}$ is an index of space grid). After that, the linear propagation step is performed with expansion coefficients in the eigenstate basis $C(t+\Delta t)=\exp \left(-i \Delta t E_{m}\right) C_{m}(t)$. Then, the back transformation from linear basis $\phi_{m}$ to coordinate basis finally gives $\psi(t+\Delta t)$. In addition to the space representation $\psi(x, y, t)$, we also compute the wave function in the momentum representation using the standard relation $\phi(\vec{p}, t)=$ $\int \psi(\vec{r}, t) \exp (-i \vec{r} \vec{p} / \hbar) d r^{2} / 2 \pi \hbar$ with a discrete Fourier transform. The time evolution computed in this way gives an approximate energy $E$ and norm $W$ conservation. Typically, we have at time $t=2000$ the variation of these integrals being $\delta W / W=0.001(0.004)$ and $\delta E / E=0.002$ (0.004) for initial state at $m=10(40)$, respectively. We return to a more detailed discussion of the accuracy of computations in Sec. V.

During the time evolution we determine the probabilities of wave function in the linear eigenmodes $\rho_{m}=\left\langle|\langle m \mid \psi\rangle|^{2}\right\rangle_{t}=$ $\left\langle\left|C_{m}\right|^{2} \mid\right\rangle_{t}$ averaged over a certain time interval. Usually, we

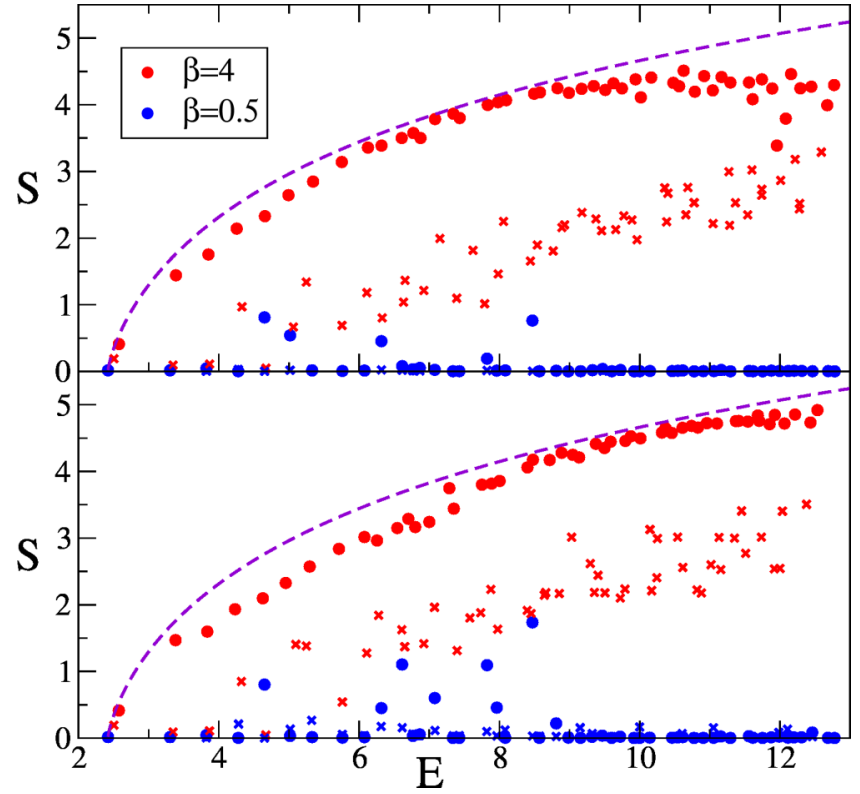

FIG. 3. Dependence of entropy $S$ on energy $E$, obtained from the GPE time evolution (2) for initial states taken as first 50 eigenstates $\phi_{m}$ of the quantum Sinai oscillator. Blue (black) and red (gray) symbols show the cases of nonlinearity $\beta=0.5$ and 4 , respectively, while circles and crosses represent the system with and without the elastic disk $r_{d}$, respectively. The entropy $S$ is computed from $\rho_{m}=\left\langle|\langle m \mid \psi\rangle|^{2}\right\rangle_{t}$ averaged over time intervals $t \in[500,1500]$ (top panel) and $t \in[1500,2500]$ (bottom panel). The dashed curve shows the theoretical thermalization ansatz of Bose-Einstein distribution (5).

choose this time interval as approximately last half (or similar to that) of the whole evolution range to obtain approximate steady-state values of $\rho_{m}$. From these averaged values we determine the entropy $S=-\sum_{m} \rho_{m} \ln \rho_{m}$. Thus, starting from different initial states, chosen as linear eigenstates $\psi_{m}$, we obtain numerically the dependence $S(E)$ which is compared with the prediction of the Bose-Einstein thermalization ansatz (5).

The comparison of numerical data with the theoretical curve obtained from (5) is shown in Fig. 3. It is clear that for small $\beta=0.5$, the nonlinear term leads to excitation of certain eigenmodes of the Sinai oscillator, but the numerical data for $S(E)$ are pretty far from the theoretical dashed curve given by the Bose-Einstein distribution (5). For the 2D oscillator without disk the excitation is significantly weaker than for the Sinai oscillator with $S(E)$ values being very far from the theory both for $\beta=0.5,4$. In contrast to that, for the GPE Sinai oscillator at $\beta=4$ our numerical data for $S(E)$ are close to the theoretical thermalization ansatz. For the time interval $t \in[500,1500]$ (Fig. 3 top panel), the obtained $S$ values for $35<m \leqslant 50(10.5<E<13)$ are somewhat below the theoretical curve. We attribute this to the fact that for large $m$, the effective amplitude of nonlinear term in (2) is reduced $|\psi|^{2} \sim 1 / x^{2} \sim 1 / E \sim 1 / \sqrt{m}$ and, hence, it takes a longer time for dynamical chaos to establish dynamical thermalization. Actually, the nonlinear energy shift as $\delta E_{\beta} \sim \beta|\psi|^{2} \sim \beta / \sqrt{m}$ and, therefore, the thermalization time $t_{T}$ should be at least proportional to $t_{T} \sim 1 / \delta E_{\beta} \sim$ $\sqrt{m} / \beta$. Indeed, at large times $t \in[1500,2500]$ (Fig. 3 bottom 


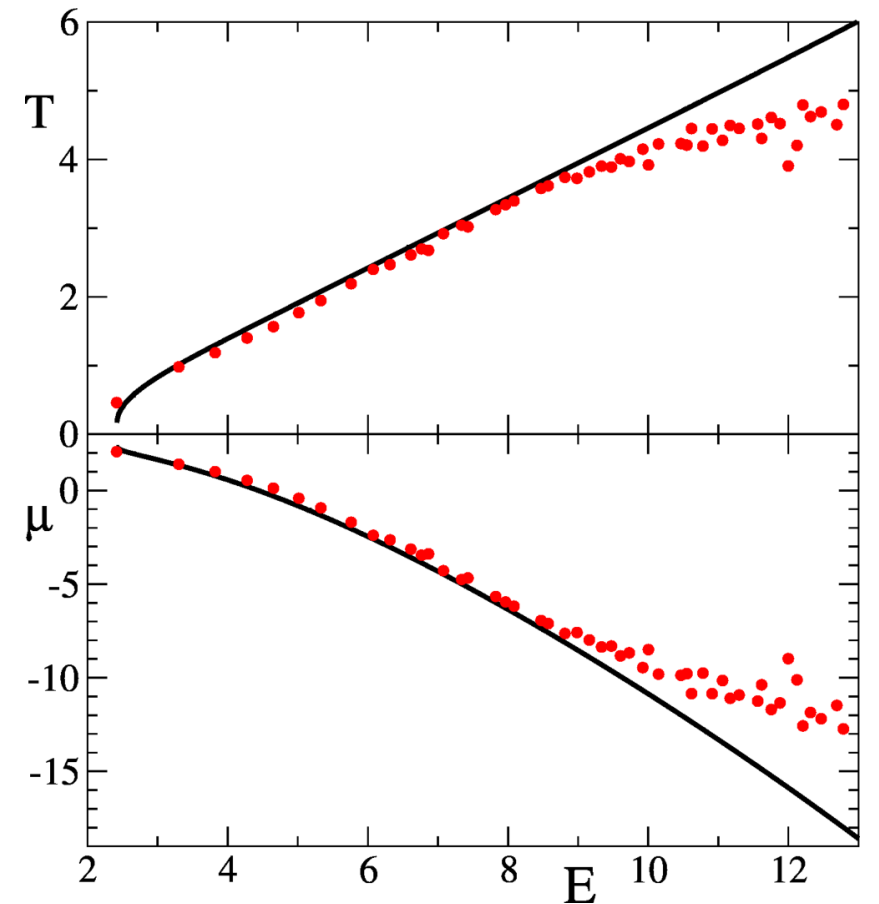

FIG. 4. Dependence of temperature $T$ and chemical potential $\mu$ on energy shown in top and bottom panels, respectively. Black curves represent the theoretical ansatz given by the Bose-Einstein distribution (5), while red circles represent numerical data $T=$ $\left[T_{1}(E)+T_{2}(S)\right] / 2$ and $\mu=\left[\mu_{1}(E)+\mu_{2}(S)\right] / 2\left(T_{1,2}\right.$ and $\mu_{1,2}$ values are computed from $E$ and $S$, respectively) for initial states given by first 50 linear eigenstates and probabilities $\rho_{m}=\left\langle|\langle m \mid \psi\rangle|^{2}\right\rangle_{t}$ averaged over interval $t \in[500,1500]$. Here, $\beta=4$.

panel) we find the values of $S$ being significantly more close to the thermalization ansatz for $35<m \leqslant 50(10.5<E<13)$. Thus, the results of Fig. 3 show the onset of dynamical thermalization for moderate values of $\beta>\beta_{c} \sim 1$.

As for the case of Bunimovich stadium [7], we expect that the thermalization border $\beta_{c} \sim 1$ (definitely $0.5<\beta_{c}<4$ ) is independent of $m$. Indeed, the nonlinear energy shift $\delta E_{\beta} \sim$ $\beta / \sqrt{m}$ and the level spacing $\Delta E \sim 1 / \sqrt{m}$ scale with $m$ in a similar way (see Fig. 2 for the dependence $E_{m}$ ) so that we can expect chaos and thermalization to be set in at $\delta E_{\beta}>$ $\Delta E$, thus leading to $\beta_{c} \sim 1$. Indeed, similar estimates have been confirmed in systems of coupled nonlinear oscillators [24,35,36].

Another confirmation of the onset of dynamical thermalization is shown in Fig. 4. According to (5) the temperature can be determined from an initial energy $E$, giving $T_{1}(E)$, or from an average value of $S$, giving $T_{2}(S)$. In a similar way we can determine $\mu_{1}(E)$ and $\mu_{2}(S)$. The dependence of average numerical values $T=\left(T_{1}+T_{2}\right) / 2, \mu=\left(\mu_{1}+\mu_{2}\right) / 2$ on energy $E$ is shown in Fig. 4, being in a good agreement with the thermalization ansatz (5). The observed deviations for $35<m \leqslant 50$ are related with the lack of sufficiently large time evolution in the simulations.

The transition from nonthermalized (quasi-integrable) regime to dynamical thermalization is also visible from the spatial probability distributions shown in Fig. 5. We start from a typical initial state $m=24$ shown in Fig. 5(b). For
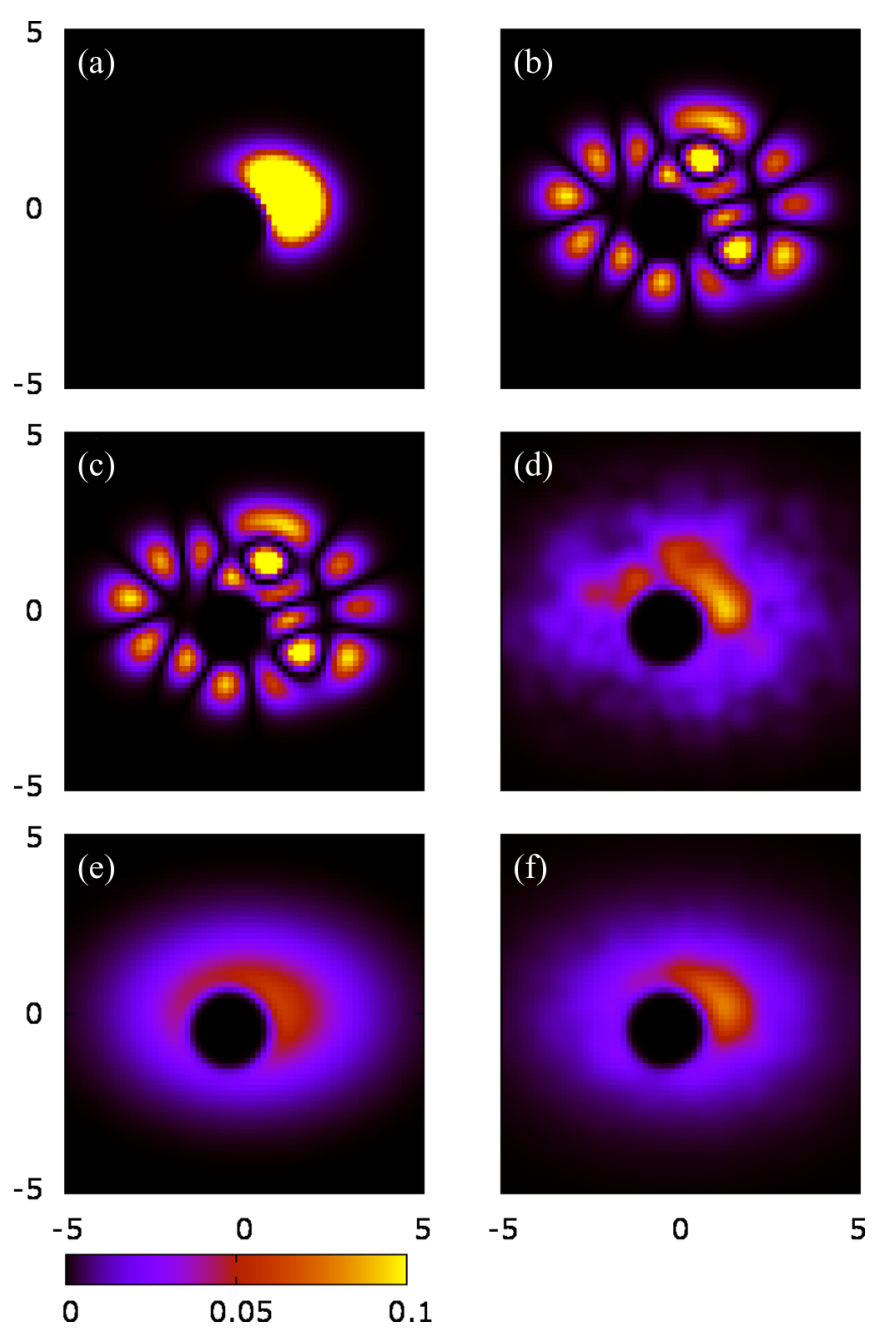

FIG. 5. Spatial probability distributions $|\psi(x, y)|^{2}$ for the GPE Sinai oscillator. Panels (a) and (b) show the linear eigenstates $m=1$ (ground state) and $m=24$ with eigenenergies $E_{1}=2.417$ and $E_{24}=$ 9.16, respectively. Panels (c) and (e) have the initial state $m=24$ of panel (b) and show the average distributions at long times with averaging over large interval $t \in[1500,2500]$ for $\beta=0.5$ and 4 , respectively. Panel (d) shows the average distribution for short time interval (snapshot) $t \in[2000,2005]$ for $\beta=4$. Panel (f) shows the theoretical thermal Bose-Einstein distribution (5) for energy $E_{24}=$ 9.16 [to be compared with panel (e)]. Probability is shown by color bar changing from zero (black) to maximum [yellow (gray)] . Numbers in horizontal and vertical axes show the scales in $x$ and $y$, respectively.

$\beta=0.5<\beta_{c}$, a snapshot distribution at $t=2000$ [Fig. 5(c)] remains very similar to the initial state showing the absence of thermalization and dominance of the initial mode. In contrast to that for $\beta=4>\beta_{c}$, a snapshot at $t=2000$ [Fig. 5(d)] shows that the distribution has a dominant component at the ground-state mode shown in Fig. 5(a). The distribution averaged over a large time interval, assumed to be close to a steady state, is shown in Fig. 5(f). It is indeed very similar to the theoretical steady-state probability distribution $\left|\psi_{s t}(x, y)\right|^{2}=\sum_{m} \rho_{m}\left|\phi_{m}(x, y)\right|^{2}$ where $\rho_{m}$ are given by the Bose-Einstein distribution (5) [see Fig. 5(e)].

The probability distributions in the momentum space $\left(p_{x}, p_{y}\right)$, corresponding to cases of Fig. 5, are shown in Fig. 6. 

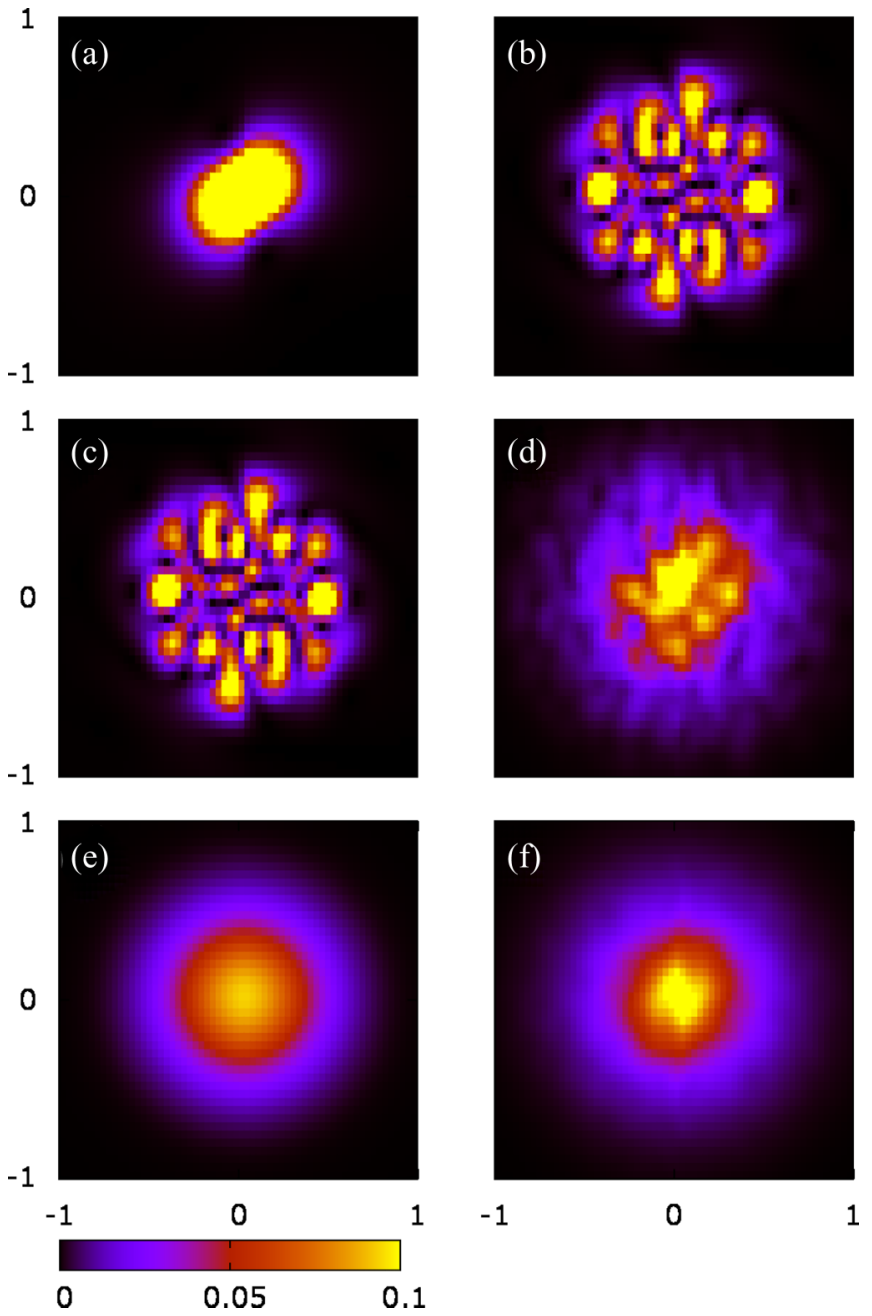

FIG. 6. Momentum probability distributions $\left|\psi\left(p_{x}, p_{y}\right)\right|^{2}$ shown for the same cases as in six panels of Fig. 5. Probability is shown by color bar changing from zero (black) to maximum [yellow (gray)]. Numbers in horizontal and vertical axes show the scales in $x$ and $y$, respectively.

These data also show a clear absence of thermalization for $\beta=0.5$ [Figs. 6(b) and 6(c)] and close similarity between the theoretical distribution [Fig. 6(e)] and average distribution [Fig. 6(f)].

Thus, the results of this section provide a good confirmation of onset of dynamical thermalization at moderate nonlinearity $\beta \approx 4>\beta_{c} \sim 1$. However, it is also important to analyze the larger scale evolution on times being larger than those considered here with $t \leqslant 2500$. This consideration is presented in the next section.

\section{LARGE TIME SCALES AND NUMERICAL METHODS}

The question about long time scale evolution of Eq. (2) requires further extensive studies with improved accuracy on numerical simulations. Indeed, at times $t>2500$ we see a tendency of probability accumulation at the ground state $\phi_{1}$ of linear system. First signs of this trend are seen in Fig. 7 showing evolution $\rho_{m}(t)$ with appearance of large values of $\rho_{1}$ at $t \approx 2300$.

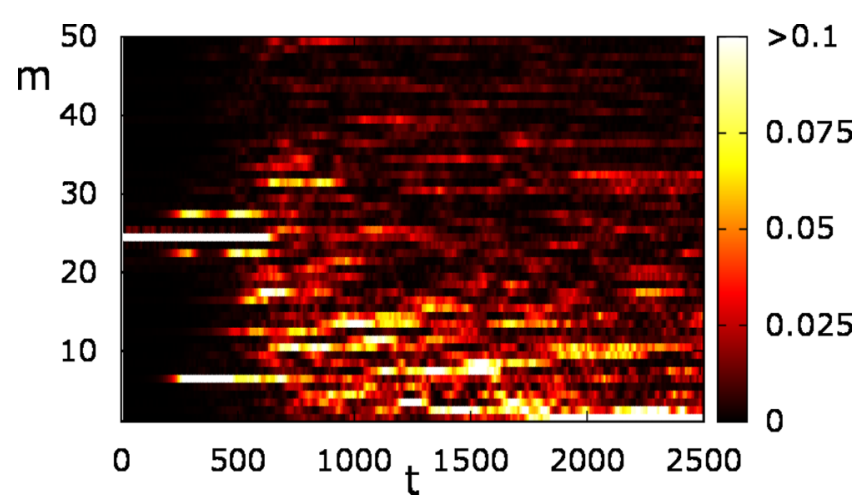

FIG. 7. Time evolution of probabilities $\rho_{m}(t)$ in the basis of linear eigenmodes for the initial state $m=24$ at $\beta=4$. The probabilities $\rho_{m}(t)$ are averaged over time $\delta t=10$ to reduce fluctuations. Color bar shows probabilities from zero (black) to maximum (white).

A more detailed view of dependence of $\rho_{m}(t)$ on time $t$ for several selected $m$ values is presented in Fig. 8 up to $t=5000$. For $m>1$, there are large fluctuations in time and it is clear that averaging on large time scales is required to obtain statistically stable values of $\rho_{m}$. For the extensive variables like energy $E$ and entropy $S$, these fluctuations are reduced and that is the reason due to which the data for the curve $S(E)$ are less fluctuating. However, for $m=1$ in Fig. 8 there is a steady growth with the apparent saturation at $t \approx 4500$ at the value $\rho_{1} \approx 0.4$ which is by a factor 10 larger than the theoretical value shown by the dashed line. The spatial distribution of probability at such large times also demonstrates a strong accumulation of probability at the ground state as it is shown in Fig. 9. A video of evolution on large times is available at [23]. A similar probability accumulation at the ground state is seen for other initial states with $\psi(t=0)=\phi_{40} ; \phi_{60}$.

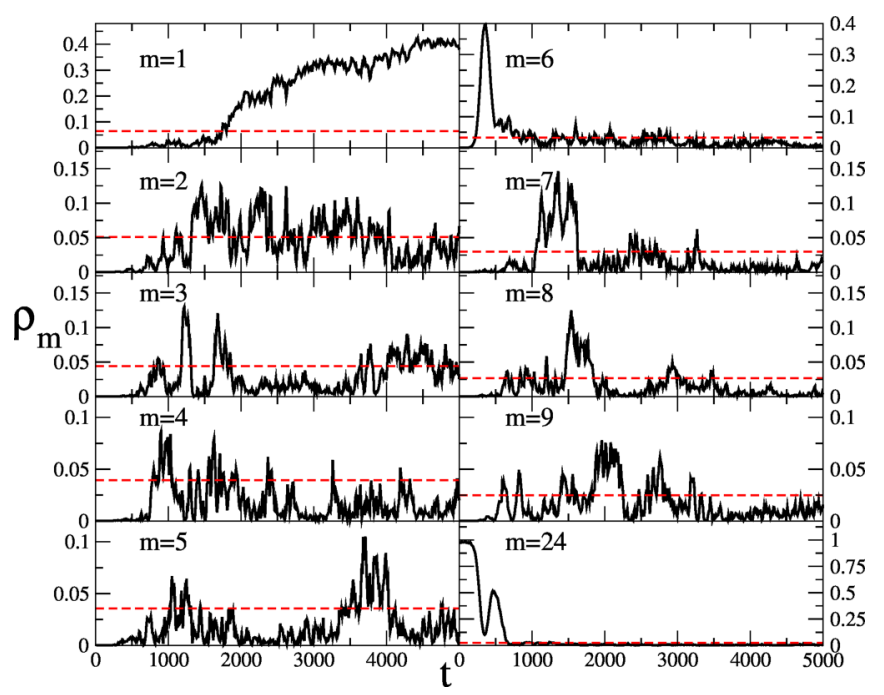

FIG. 8. Time evolution of probabilities $\rho_{m}(t)$ in the basis $f$ of linear eigenmodes for the initial state $m=24$ at $\beta=4$. The probabilities $\rho_{m}(t)$ are averaged over time $\delta t=10$ to reduce fluctuations. 10 panels show $\rho_{m}(t)$ for $m=1,2,3,4,5,6,7,8,9$ and $m=24$; red dashed lines show the theoretical values of $\rho_{m}$ from the thermalization ansatz of Bose-Einstein distribution (5). 


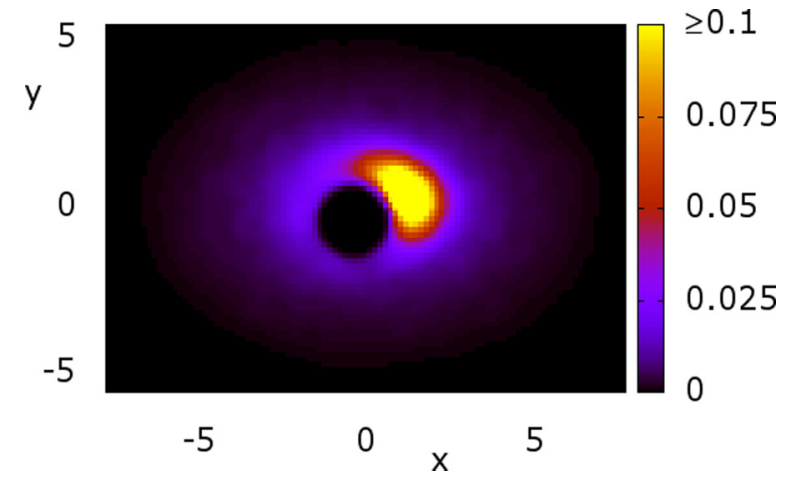

FIG. 9. Long time average probability distribution $\left\langle|\psi(x, y)|^{2}\right\rangle_{t}$ shown in coordinate space. The evolution starts from the initial linear eigenstate $m=24$ (see Fig. 5), the average is done over the time interval $t \in[4000,5000]$; here $\beta=4$ (to compare with panels of Fig. 5 with same colors). Numbers in horizontal and vertical axes show the scales in $x$ and $y$, respectively.

We note that we had no such accumulation of probability in the ground state in the studies of GPE Bunimovich billiard [7].

We also considered the time evolution for a different initial state taken as initial coherent wave packet centered at certain position $\left(x_{0}, y_{0}\right)$ (of course the packet is only approximately Gaussian since the probability is zero at the disk border). The initial distribution and snapshots at a few moments of time are shown in Figs. 10(a)-10(c). The time evolution video is available at [23] for $\beta=0$ and 4 on short and long time scales. The probability averaged over time interval $t \in[4000,5000]$ is shown in Figs. 10(f) and 10(d) $\beta=0$ and 4, respectively. The case with interactions shows a tendency of accumulation of probability at low- $m$ modes in a qualitative agreement with the thermal ansatz distribution shown in Fig. 10(e). However, it is visible that the GPE case has a larger probability on low-energy modes $m$. In contrast, the case with $\beta=0$ [Fig. 10(f)] has large probability in initial high modes $m$.

The average probability distributions over linear modes are shown at large times in Fig. 11. For the initial state in a form of coherent packet there is a clear displacement of highest probabilities from initial energies $E_{m} \approx 14.7$ to modes with $m=1,2,4$. In this case, there is no strong accumulation of probability at the ground state. In fact, the average value of $\rho_{1} \approx 0.22$ is comparable with the thermalization ansatz value. Also for this state with energy $E \approx 14.7$ we find numerically the average entropy value $S=4.96$ being close to the theoretical value of (5) with $S=5.47$. However, the fluctuations of average $\rho_{m}$ probabilities are too strong and the comparison with the thermalization ansatz curve is only qualitative. For the initial state with $\phi_{24}$, the probability $\rho_{1}$ is significantly larger then the theoretical value (by a factor $10)$, then the decay of $\rho_{m}$ with $E_{m}$ approximately follows the thermalization ansatz but also the fluctuations are large. We think that the fluctuations are larger for the case of coherent state due to a larger number of initially excited linear eigenmodes compared to the case with $\psi(t=0)=\phi_{24}$. Also, the initial energy of the coherent state is $E=14.71$ being larger than energy of $m=24$ with $E=9.16$ and hence longer
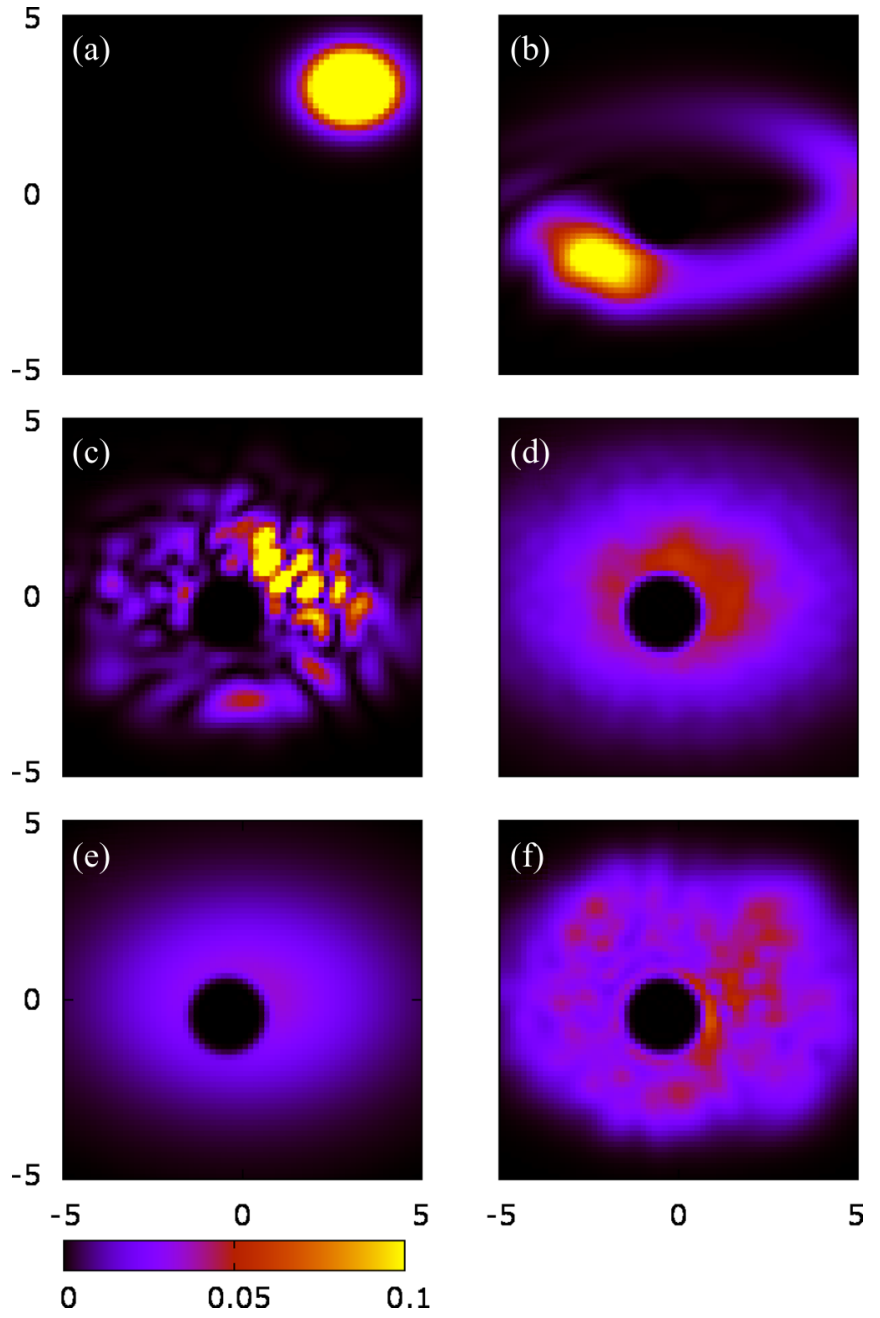

FIG. 10. Space probability distributions $|\psi(x, y)|^{2}$ for the case of an initial state given by a coherent wave packet centered at $\left(x_{0}, y_{0}\right)=(3,3)$ (and zero probabilities on the disk). Panel (a) shows the initial-state distribution with $E=14.707$; panels (b) and (c) show distributions with $\beta=4$ at $t=2.9$ and $t=8$, respectively. Long time averages over the interval $t \in[4000,5000]$ are shown in panels (d) and (f) for $\beta=4$ and 0 (linear evolution), respectively. Panel (e) shows the theoretical space probability from the Bose-Einstein distribution (5) for energy $E=14.707$. Numbers in horizontal and vertical axes show the scales in $x$ and $y$, respectively.

times are required for complete thermalization of the coherent state.

To check the validity of numerical integration for large time scales we performed a number of checks shown in Figs. 12 and 13. With this aim we varied the integration time step $\Delta t$, the number of lattice points $N_{s}$ in the coordinate space $\left(-x_{\min }, x_{\max } ;-y_{\min }, y_{\max }\right)$, and the spatial range of the lattice determined by these $\min$ and $\max$ values of $x, y$. The integration scheme gives a slow decrease of norm $W$ and energy $E$ with time indicating that there is some effective dissipation induced by numerical integration. These checks show that the most sensitive parameter is the number of eigenstates $N_{e}$ used in the transformation matrix $A_{j, m}$ from coordinate space to linear eigenmodes. Up to time $t \approx 800$, 


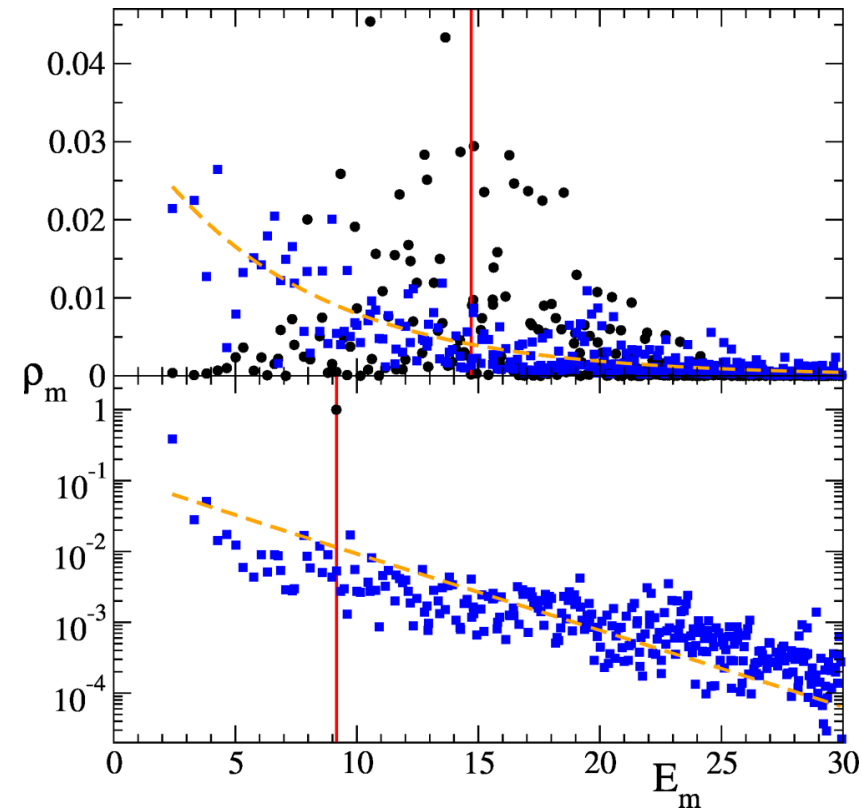

FIG. 11. Probability $\rho_{m}$ vs energy of linear eigenstate $E_{m}$. Top panel shows the case of initial state given by a coherent state localized at $\left(x_{0}, y_{0}\right)=(3,3)$ from Fig. 10; bottom panel shows the case of initial linear eigenstate $m=24$ (semilogarithmic scale). Black circles and blue squares show $\rho_{m}$ for initial state and for long time average with $\beta=4$ and $t \in[4000,5000]$, respectively. Solid red line shows the total energy, while dashed orange line represents the theoretical distribution $\rho_{m}$ (5) for corresponding energy $E=14.71$ and 9.16 (top and bottom panels, respectively).

all numerical curves in Fig. 13 give the same results showing the validity of numerical integration. However, for $N_{e}=1000$ the nonconservation of $W$ and $E$ becomes significant at large times, giving significantly different values of $\rho_{1}, \rho_{4}$ at $t \approx 1000-1500$ for $N_{e}=1000$ and 2000 . Thus, we use the numerical parameters with $N_{e}=2000$ for all results presented in above Figs. 1-11 since this choice provides the best conservation of $W$ and $E$.

The above verification shows that our integration scheme introduces a hidden intrinsic effective dissipation and due to this reason we consider that this can be the reason of probability accumulation at the ground state (or a few states with low- $m$ values) at large times. Due to this reason, we consider that our numerical results are reliable only up to finite times $t \leqslant t_{\text {num }} \approx$ 2500 . We believe that better symplectic integration schemes should be developed to extend numerical studies for larger time scales.

Finally, in Fig. 14 we present data for the variation of entropy $S$ with the nonlinear parameter $\beta$ obtained for different initial states $m$. The data are averaged over a certain time interval. The ratio of numerical $S$ values to the expected theoretical values $S_{\text {theo }}$, given by the Bose-Einstein ansatz (5), has a sharp growth at a certain $\beta=\beta_{c} \approx 1.5$ indicating a thermalization transition at this $\beta_{c}$. At the same time, there is a certain spreading of curves which we attribute to fluctuations and the fact that longer time intervals are required for larger$m$ values where nonlinear frequencies become smaller (see discussion of this point in the sections above). Thus, due to a

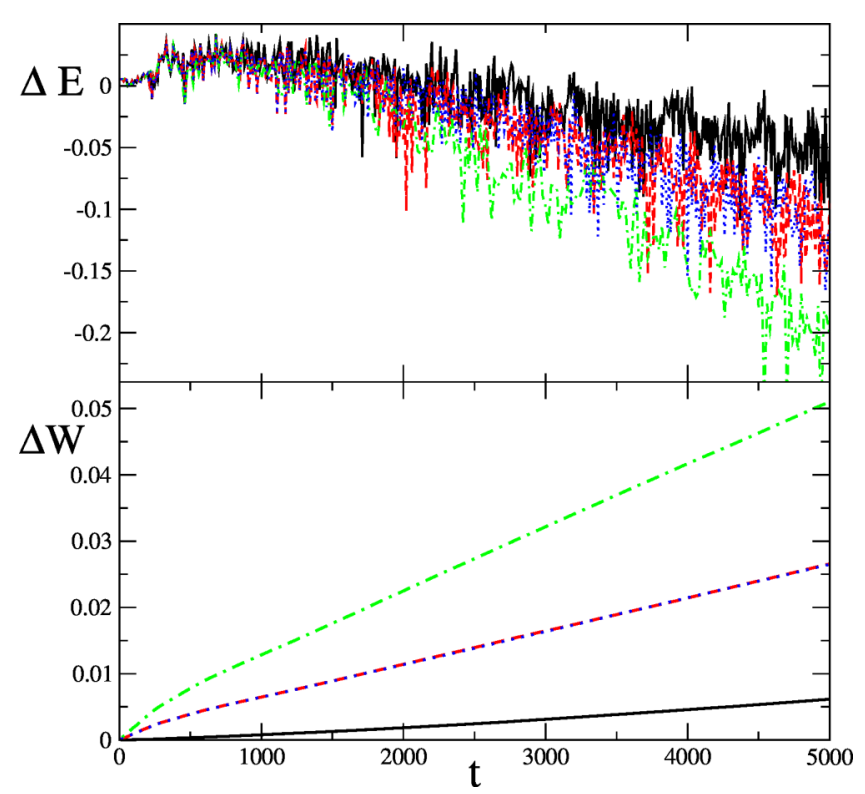

FIG. 12. Time evolution of energy and norm conservation for different parameters of numerical simulations with the initial linear eigenstate $m=24$ and $\beta=4$. Top panel and bottom panel show the evolution of $\Delta E=E-E_{24}$ and $\Delta W=1-\sum_{m} \rho_{m}$. The simulation parameters are $N_{e}=2000, \Delta t=0.01$ and number of spatial lattice of $N_{s}=201 \times 141$ points in the rectangle given by $\left(x_{\min }, y_{\min }\right)=(-\sqrt{234} \approx-15.3,-\sqrt{117} \approx-10.82)$ and $\left(x_{\max }, y_{\max }\right)=(\sqrt{234} \approx 15.3, \sqrt{117} \approx 10.82)$ (black solid curves); $N_{e}=1000, \Delta t=0.01$ with $N_{s}=201 \times 141$ (red dashed curves); $N_{e}=1000, \Delta t=0.005$ with $N_{s}=201 \times 141$ (green dotted-dashed curves); $N_{e}=1000, \Delta t=0.01$ with $N_{s}=361 \times 255$ lattice points in the same region (blue dotted curves); red and blue curves practically coincide in the bottom panel.

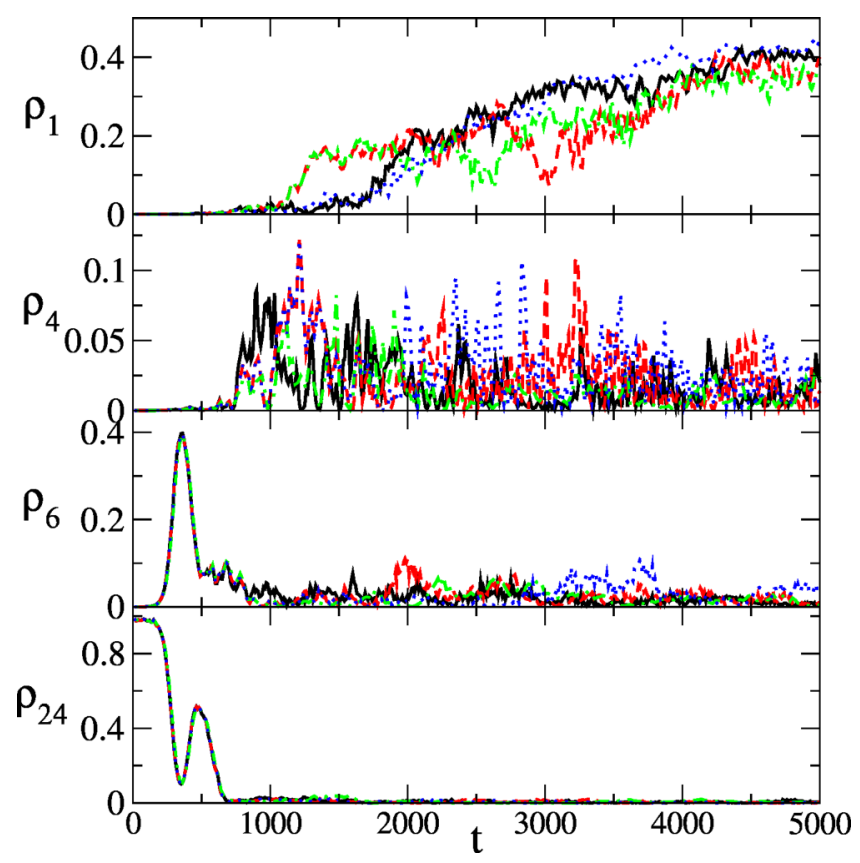

FIG. 13. Time evolution of probabilities $\rho_{m}(t)$ for parameters of Fig. 12 shown for $m=1,4,6,24$ with the same colors as in Fig. 12. 


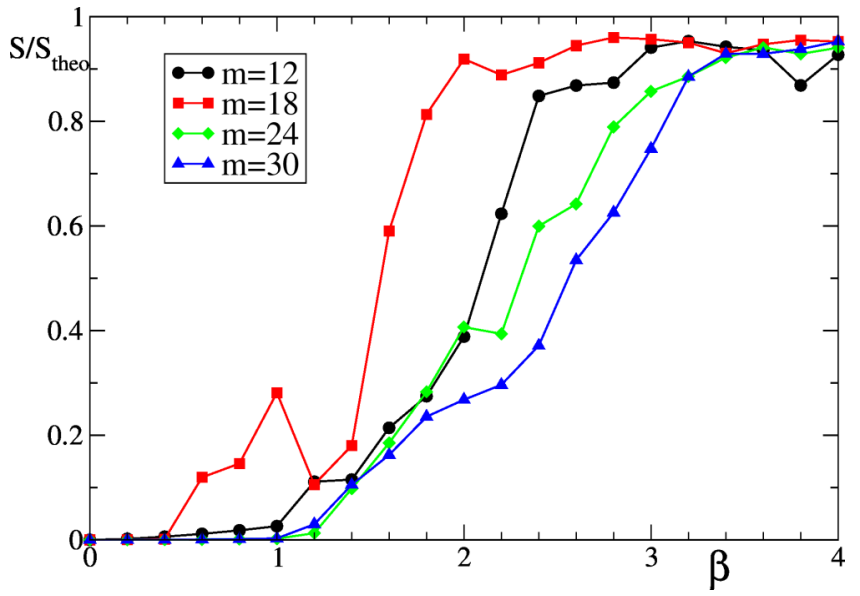

FIG. 14. Entropy $S / S_{\text {theo }}$ as a function of nonlinear parameter $\beta$. The entropy $S$ is computed numerically in linear basis with probability average $\rho_{m}$ over the time interval $t \in[1000-2000]$. Initial states are linear eigenstates with $m=12,18,24$, and 30 represented with circles, squares, diamonds, and triangles, respectively. The values of $S$ are normalized by the theoretical values $S_{\text {theo }}$ given by the Bose-Einstein ansatz (5) for the corresponding initial state $m$.

restricted time of our numerical simulations we cannot exclude that instead of a sharp transition to thermalization, there is a crossover in a certain $\beta$ interval. In any case, the data of Fig. 14 show that for $\beta>3$ the values of entropy $S$ become close to the expected theoretical values with $S / S_{\text {theo }}=1$. Thus, we conclude that the dynamical thermalization is reached for $\beta>2-3$.

\section{DISCUSSION}

The results of this work demonstrate the emergence of dynamical thermalization of BEC described by the GPE equation of wave-function time evolution in Sinai-oscillator trap. The classical dynamics of rays in such a trap is chaotic and the quantum properties of this system in absence of interactions are described by well-known results of the field of quantum chaos. The dynamical thermalization appears above a certain critical strength of interaction $\beta>\beta_{c}$. In this thermalized phase, the probability distribution $\rho_{m}$ over linear eigenmodes (at $\beta=0$ ) is well described by the standard statistical Bose-Einstein distribution. We stress that the dynamical thermalization appears in a completely isolated system without any external noise. We point that this thermal distribution is drastically different from energy equipartition over modes which is usually expected for nonlinear oscillator lattices, including the Fermi-Pasta-Ulam problem, leading to the ultraviolet catastrophe. Thus, our results show that the energy is redistributed only over certain low-energy modes and that there is no energy flow to high-energy modes. We think that this result may have interesting implications to the dynamical consideration of Kolmogorov turbulence which assumes the presence of energy flow from large (low-energy) to small (high-energy) spatial scales in presence of noise [29,30]. Our results indicate that in absence of noise, such energy flow can be absent due to absence of energy equipartition. Of course, further investigations of this system in numerical simulations on larger time scales are highly desirable in view of numerical difficulties discussed in the previous section.

The trap configuration considered here had been already realized experimentally in 3D [1] and we believe that further experimental investigations of dynamical thermalization in the Sinai-oscillator trap are accessible for modern experiments with cold atoms and BEC. The variation of interaction strength between atoms by means of Feshbach resonance should be able to detect a transition from quasi-integrable phase at $\beta<\beta_{c}$ to the phase of dynamical thermalization at $\beta>\beta_{c}$. The case of lithium- 6 atoms, where the interactions can be changed in a broad range (see e.g. [37]), can be a good test bed for the studies of dynamical thermalization in the Sinai-oscillator trap and fundamental origins of thermalization in isolated systems.

\section{ACKNOWLEDGMENTS}

We thank P. Chapovsky and D. Guéry-Odelin for useful discussions of cold-atom physics.
[1] K. B. Davis, M.-O. Mewes, M. R. Andrews, N. J. van Druten, D. S. Durfee, D. M. Kurn, and W. Ketterle, Bose-Einstein Condensation in a Gas of Sodium Atoms, Phys. Rev. Lett. 75, 3969 (1995).

[2] J. A. Anglin and W. Ketterle, BoseEinstein condensation of atomic gases, Nature (London) 416, 211 (2002).

[3] W. Ketterle, Nobel lecture: When atoms behave as waves: BoseEinstein condensation and the atom laser, Rev. Mod. Phys. 74, 1131 (2002).

[4] F. Dalfovo, S. Giorgini, L. P. Pitaevskii, and S. Stringari, Theory of Bose-Einstein condensation in trapped gases, Rev. Mod. Phys. 71, 463 (1999).

[5] L. Pitaevskii and S. Stringari, Bose-Einstein Condensation (Oxford University Press, Oxford, 2003).

[6] Ya. G. Sinai, Dynamical systems with elastic reflections. Ergodic properties of dispersing billiards, Usp. Mat. Nauk 25, 141 (1970) [Russian Math. Surveys 25, 137 (1970)].
[7] L. Ermann, E. Vergini, and D. L. Shepelyansky, Dynamical thermalization of Bose-Einstein condensate in Bunimovich stadium, Europhys. Lett. 111, 50009 (2015).

[8] O. Bohigas, M. J. Giannoni, and C. Schmit, Characterization of Chaotic Quantum Spectra and Universality of Level Fluctuation Laws, Phys. Rev. Lett. 52, 1 (1984).

[9] E. Wigner, Random matrices in physics, SIAM Rev. 9, 1 (1967).

[10] F. Haake, Quantum Signatures of Chaos (Springer, Berlin, 2010).

[11] H.-J. Stockmann, Microwave billiards and quantum chaos, Scholarpedia 5, 10243 (2010).

[12] L. D. Landau and E. M. Lifshitz, Statistical Physics (Wiley, New York, 1976).

[13] S. B. Kuksin, Hamiltonian PDEs, in Handbook of Dynamical Systems, edited by B. Hasselblatt and A. Katok (Elsevier, Amsterdam, 2006), Vol. 1B, p. 1087. 
[14] L. H. Eliasson, B. Grebert, and S. B. Kuksin, KAM for the non-linear Beam equation 2: A normal form theorem, arXiv:1502.02262 (2015).

[15] M. Planck, Ueber das gesetz der energieverteilung im normalspectrum, Ann. Phys. (Berlin) 309, 553 (1901).

[16] E. Fermi, J. Pasta, S. Ulam, and M. Tsingou, Studies of non linear problems, Los Alamos Report No. LA-1940, 1955 (unpublished).

[17] E. Fermi, Collected Papers, Vol. 2 (University of Chicago Press, Chicago, 1965).

[18] D. K. Campbell, P. Rosenau, and G. Zaslavsky (eds.), A Focus Issue on "The Fermi- Pasta-Ulam problem: The first 50 years," Chaos 15, 015101 (2005).

[19] The Fermi-Pasta-Ulam Problem, Lecture Notes in Physics, edited by G. Gallavotti (Springer, Berlin, 2008), p. 728.

[20] B. V. Chirikov, A universal instability of many-dimensional oscillator systems, Phys. Rep. 52, 263 (1979).

[21] A. J. Lichtenberg and M. A. Lieberman, Regular and Chaotic Dynamics (Springer, Berlin, 1992).

[22] E. Vergini and M. Saraceno, Calculation by scaling of highly excited states of billiards, Phys. Rev. E 52, 2204 (1995).

[23] Sinai oscillator web page: http://www.quantware.ups-tlse.fr/ QWLIB/sinaioscillator

[24] D. L. Shepelyansky, Delocalization of Quantum Chaos by Weak Nonlinearity, Phys. Rev. Lett. 70, 1787 (1993).

[25] A. S. Pikovsky and D. L. Shepelyansky, Destruction of Anderson Localization by a Weak Nonlinearity, Phys. Rev. Lett. 100, 094101 (2008)

[26] I. Garcia-Mata and D. L. Shepelyansky, Delocalization induced by nonlinearity in systems with disorder, Phys. Rev. E 79, 026205 (2009).
[27] S. Fishman1, Y. Krivolapov, and A. Soffer, The nonlinear Schrödinger equation with a random potential: Results and puzzles, Nonlinearity 25, R53 (2012).

[28] T. V. Laptyeva, M. V. Ivanchenko, and S. Flach, Nonlinear lattice waves in heterogeneous media, J. Phys. A: Math. Theor. 47, 493001 (2014).

[29] V. E. Zakharov, V. S. L'vov, and G. Falkovich, Kolmogorov Spectra of Turbulence (Springer, Berlin, 1992).

[30] S. Nazarenko, Wave Turbulence (Springer, Berlin, 2011).

[31] S. Nazarenko, M. Onorato, and D. Proment, Bose-Einstein condensation and Berezinskii-Thouless transition in the twodimensional nonlinear Schrödinger model, Phys. Rev. A 90, 013624 (2014).

[32] K. Fujimoto and M. Tsubota, Bogoliubov-wave turbulence in Bode-Einstein condensates, Phys. Rev. A 91, 053620 (2015).

[33] D. L. Shepelyansky, Kolmogorov turbulence, Anderson localization and KAM integrability, Eur. Phys. J. B 85, 199 (2012).

[34] M. Mulansky, K. Ahnert, A. Pikovsky, and D. L. Shepelyansky, Dynamical thermalization of disordered nonlinear lattices, Phys. Rev. E 80, 056212 (2009).

[35] L. Ermann and D. L. Shepelyansky, Quantum Gibbs distribution from dynamical thermalization in classical nonlinear lattices, New J. Phys. 15, 12304 (2013).

[36] B. V. Chirikov and D. L. Shepelyansky, Dynamics of some homogeneous models of classical Yang-Mills fields, Yd. Fiz. 36, 1563 (1982) [Sov. J. Nucl. Phys. 36, 908 (1982)].

[37] T. Bourdel, L. Khaykovich, J. Cubizolles, J. Zhang, F. Chevy, M. Teichmann, L. Tarruell, S. J. J. M. F. Kokkelmans, and C. Salomon, Experimental Study of the BEC-BCS Crossover Region in Lithium 6, Phys. Rev. Lett. 93, 050401 (2004). 